\title{
Magnon crystals and magnetic phases in a kagome-stripe antiferromagnet
}

\author{
S. Acevedo, ${ }^{1}$ C. A. Lamas $\odot,{ }^{1}$ M. Arlego, ${ }^{1}$ and P. Pujol $^{2}$ \\ ${ }^{1}$ IFLP-CONICET, Departamento de Física, Facultad de Ciencias Exactas, Universidad Nacional de La Plata, \\ C.C. 67, 1900 La Plata, Argentina \\ ${ }^{2}$ Laboratoire de Physique Thèorique-IRSAMC, Centre National de la Recherche Scientifique and Universitè de Toulouse, \\ UPS, Toulouse F-31062, France
}

(Received 29 August 2019; revised manuscript received 10 October 2019; published 27 November 2019)

\begin{abstract}
In this paper we analyze the magnetization properties of an antiferromagnetic kagome-stripe lattice, motivated by the recent synthesis of materials exhibiting this structure. By employing a variety of techniques that include numerical methods such as density-matrix renormalization-group and Monte Carlo simulations, as well as analytical techniques such as perturbative low-energy effective models and exact solutions, we characterize the magnetization process and magnetic phase diagram of a kagome-stripe lattice. The model captures a variety of behaviors present in the two-dimensional kagome lattice, which are described here by analytical models and numerically corroborated. In addition to the characterization of semiclassical intermediate plateaus, it is worth noting the determination of an exact magnon crystal phase which breaks the underlying symmetry of the lattice. This magnon crystal phase generalizes previous findings.
\end{abstract}

DOI: 10.1103/PhysRevB.100.195145

\section{INTRODUCTION AND MODEL}

The interplay between geometric frustration and quantum fluctuations enhanced by low dimensionality results in a rich behavior and variety of exotic phases such as spin liquids, the experimental identification of which, despite theoretical description [1-4], presents great challenges [5].

A paradigmatic case is the spin-1/2 kagome lattice antiferromagnet, which finds experimental realization in several compounds, such as the Herbertsmithite $\mathrm{ZnCu}_{3}(\mathrm{OH})_{6} \mathrm{Cl}_{2}$ [6], $\alpha$-vesignieite $\mathrm{BaCu}_{3} \mathrm{~V}_{2} \mathrm{O}_{8}(\mathrm{OH})_{2}$ [7], and $\left[\mathrm{NH}_{4}\right]_{2}\left[\mathrm{C}_{7} \mathrm{H}_{14} \mathrm{~N}\right]\left[\mathrm{V}_{7} \mathrm{O}_{6} \mathrm{~F}_{1} 8\right]_{5}$ [8]. The spin-1/2 kagome lattice antiferromagnet has been proposed to exhibit a spinliquid ground state, although this aspect has not been fully clarified yet [9-13].

Another source of exotic phases is the Bose-Einstein condensate (BEC), where a macroscopic number of bosons configure a single-particle quantum state [14]. In antiferromagnetic insulators, the magnetic excitations are usually bosonic magnons, the interaction of which with the underlying crystalline lattice can lead to a rich phenomenology, including BECs $[15,16]$.

The presence of an external magnetic field incorporates an extra degree of freedom that favors the emergence of a variety of behaviors and phases. The simple image of a magnetization curve that grows gradually with the magnetic field until it reaches saturation, in frustrated quantum systems, can became considerably more complex.

On the one hand, flat regions, called plateaus, can emerge where magnetization remains constant at a certain fraction of saturation, in a range of applied magnetic fields [17]. Plateaus can have a classical origin, in the sense that they can be described in terms of relative orientations of classical spins [18]. However, there are plateaus that only admit a quantum description, in terms of elemental magnon or spinon excitations [19].
Another ingredient is the appearance of jumps in the magnetization curve, due to different mechanisms, such as first-order transitions between classical [20] or quantum states or BECs of a purely quantum nature [21].

Interacting magnons in a BEC can be localized on certain places of the lattice due to frustration and crystallize through a superfluid-insulator transition, giving rise to a "magnon crystal" phase $[21,22]$.

Magnon crystal phases are present in a variety of frustrated magnets. In particular, the spin-1/2 kagome lattice antiferromagnet magnon crystal phases have been predicted below saturation [23,24] and found experimentally in the synthetic Cdkapellasite at very high magnetic fields where the magnons localize on the hexagon of the kagome lattice [25].

The generality and richness of behaviors described before is also expected in a reduced geometry. This aspect is further enhanced by the recent synthesis of two tellurite sulfates $A_{2} \mathrm{Cu}_{5}\left(\mathrm{TeO}_{3}\right)\left(\mathrm{SO}_{4}\right)_{3}(\mathrm{OH})_{4}$ with $A=\mathrm{Na}$ or $\mathrm{K}$ [26]. In these compounds, the topological structure of $\mathrm{Cu}^{+2}$ ions exhibits a one-dimensional kagome-stripe lattice. The experimental determination of the crystalline structure shows that the kagome stripe is distorted, showing five different $\mathrm{Cu}-\mathrm{Cu}$ distances, as indicated in Fig. 2(c) of Ref. [26]. In addition, the study of these compounds suggests an antiferromagnetic behavior and indicates the existence of antiferromagnetic order and some field induced magnetic transitions.

Motivated by the mentioned compounds and phenomenology we study the Heisenberg model on the kagome-stripe lattice presented in Fig. 1, in the presence of an external magnetic field:

$$
H=\sum_{\langle i, j\rangle} J_{i, j} \vec{S}_{i} \cdot \vec{S}_{j}-h \sum_{i} \vec{S}_{i}^{z} .
$$

We start with a five-spin unit cell in the lattice and four different magnetic couplings $J_{i, j}$ as schematized in Fig. 1. Note 


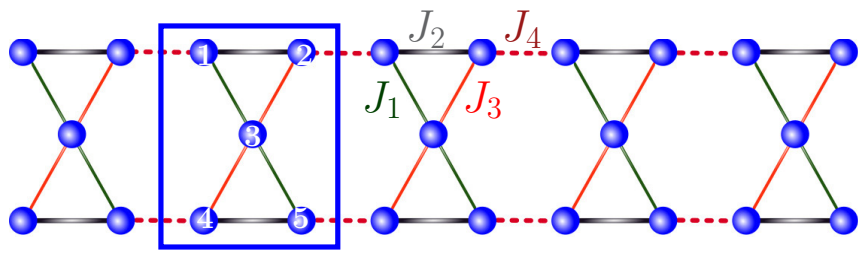

FIG. 1. Geometry of the kagome-stripe lattice considered in this paper. The four antiferromagnetic couplings $J_{i}, i=1, \ldots, 4$ can be different to model the lattice distortion. The unit cell is framed with blue lines and presents geometrical frustration. The white integers $1, \ldots, 5$ are used to index the sites within the unit cell.

that, although the material involves five different couplings, in this paper we consider a space of four couplings. In this way an extra reflection symmetry is maintained, which simplifies the analysis, without losing the complexity of the unit cell of the material. This is also justified because it is not intended to describe properly the material.

Throughout this paper we will concentrate on different variants and limiting cases of the model to analyze the possible semiclassical and quantum phases that may be present in this system, and that are of potential interest for the description of the actual materials. In this context we would like to highlight the study by Morita et al. [27], who analyze the structure of magnetization curves in the subspace $J_{1}=J_{3}$ (Fig. 1) of our model.

A central aspect of this paper is the analysis of the structure of the magnetization curves of the model. In this context the
Oshikawa-Yamanaka-Affleck (OYA) theorem [28] provides the necessary condition for the presence of magnetization plateaus as

$$
N S(1-m)=\text { integer, }
$$

where $N$ is the number of spins in the ground-state unit cell presenting spatial periodicity and $m=\frac{M}{M_{\text {sat }}}$ is the normalized magnetization per site. According to (2), if the translational symmetry of the lattice is preserved in the ground state $(N=$ 5), the magnetization curve may have plateaus at $m=1 / 5$ and $3 / 5$. On the other hand, the emergence of plateaus at different magnetization values is an indication of a spontaneous breaking of the translation symmetry in the ground state.

In this paper we will explore both variants of phases that respect or break the underlying symmetry of the lattice, as well as their semiclassical or quantum character. To this end we will use a variety of analytical techniques that will allow us to describe the different emerging plateaus in semiclassical terms or by means of low-energy effective models, complemented with numerical methods. The result is a single model with a rich structure of phases, exhibiting semiclassical signatures, as well as truly quantum aspects, as a generalized crystal magnon phase.

The paper is organized as follows. In Sec. II we study the presence of semiclassical (Ising-like) plateaus in the magnetization curve, and we present the phase diagram in a representative subset of the parameter space. For this we employ density-matrix renormalization-group (DMRG) calculations. The main part of this section concerns the analysis
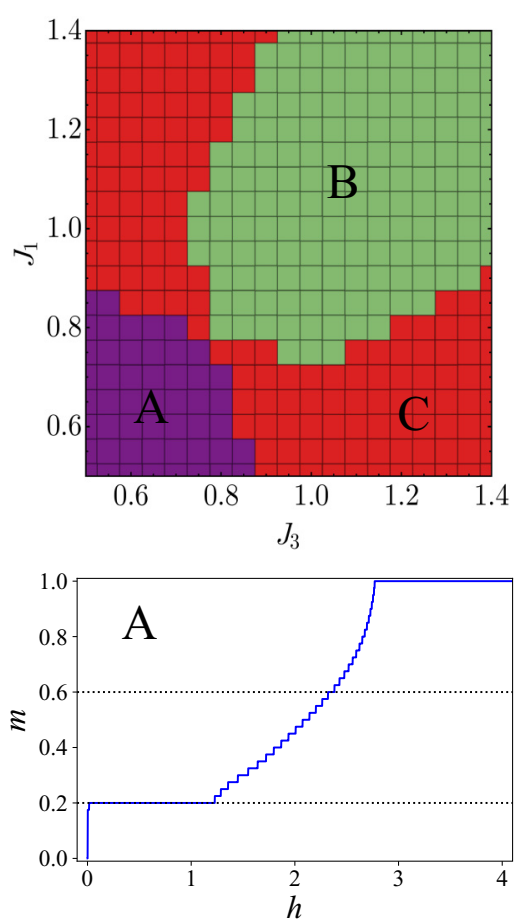
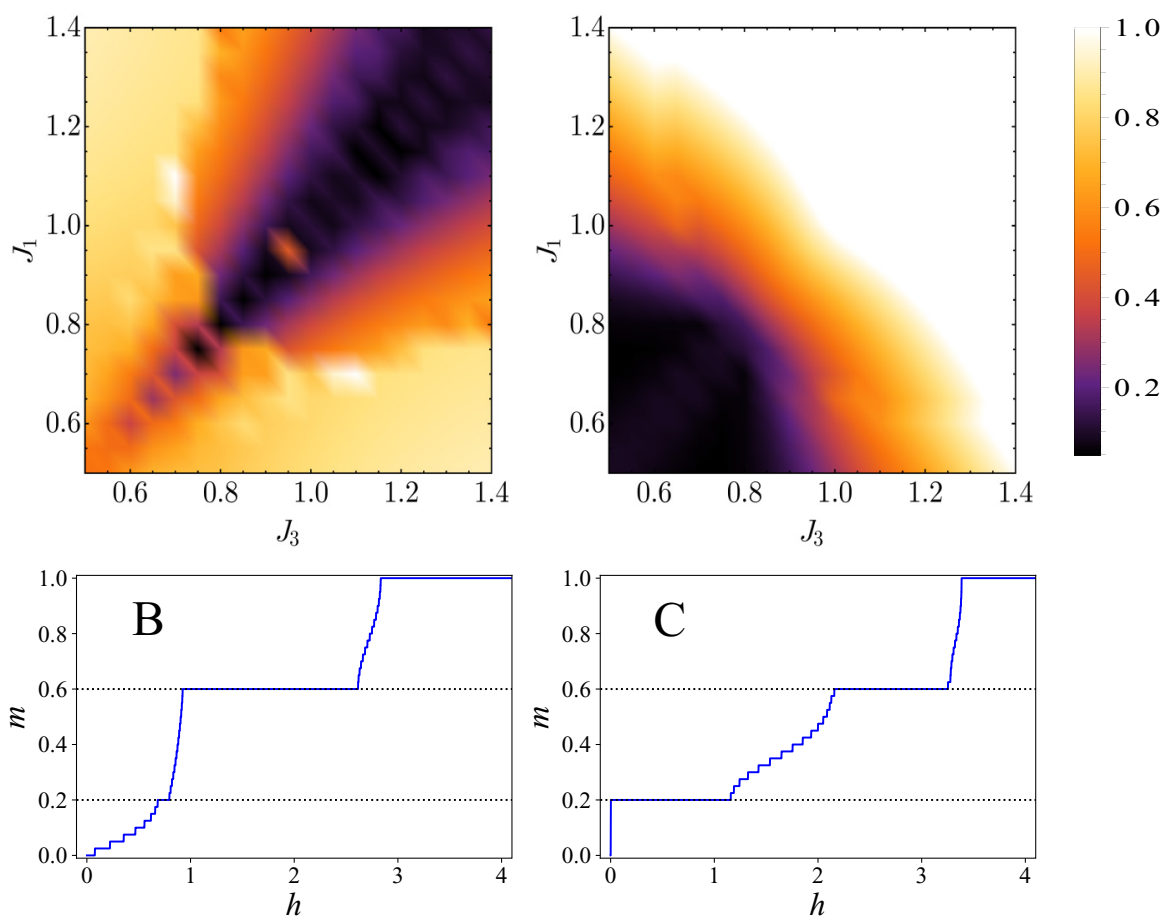

FIG. 2. Phase diagram of the model in a representative sector of the $J_{3}-J_{1}$ plane at $J_{2}=1$ and $J_{4}=0.8$, evaluated by means of DMRG for a stripe with 180 spins (top left panel). The magnetic phases A, B, and C are defined by the presence of magnetization plateaus with $m=1 / 5,3 / 5$, and both $1 / 5$ and $3 / 5$, respectively. Magnetization curves representative of each phase are depicted in bottom panels. We selected $\left(J_{3}, J_{1}\right)=\left(\frac{7}{15}, \frac{2}{3}\right),\left(\frac{11}{5}, 2\right)$, and $\left(1, \frac{2}{3}\right)$ for A, B, and C phases, respectively. The plateaus widths, in units of $h$, corresponding to $m=1 / 5(m=3 / 5)$ are shown in top middle (right) panels. 
of the origin of the semiclassical plateaus. This is rooted to the classical kagome-stripe lattice model and finally to the underlying Ising order of the unit cell. To this end, we study this limiting case explicitly in several subsections, via Monte Carlo simulations and low-energy effective models starting from the strong plaquette (unit-cell) limit. The low-energy model shows that semiclassical plateaus (in particular $m=$ $3 / 5$ ) are perturbatively connected with this limit, completing the analysis of nonbreaking lattice symmetry phases of this section.

In Sec. III we study phases with spontaneously broken translational symmetry, in which a periodic structure of localized magnons emerges, and in particular at $m=4 / 5$. For a range of values within the parameter space we find exact ground states of magnon crystal phases [21]. All these results are complemented with numerical DMRG determination of magnetization curves.

\section{SEMICLASSICAL PLATEAUS}

In this section we analyze the phases of semiclassical plateaus at $m=1 / 5$ and $3 / 5$. These phases preserve the lattice translational symmetry, according to the OYA theorem given by (2). To determine the extension of the phases we have evaluated the magnetization as a function of the applied magnetic field for several values of the couplings, by means of DMRG calculations for large stripes (200 spins). The DMRG computations were performed with the open source code ALPS [29]. For the calculations, we kept up to 500 states throughout the paper, which proved to be enough to achieve the required precision.

Due to the size of the parameter space, here we illustrate our results in a sector of the $J_{3}-J_{1}$ plane at $J_{2}=1$ and $J_{4}=$ 0.8 . This subspace captures regions where the plateaus exist separately or coexist.

The results are presented in the phase diagram of Fig. 2 (top left), where the $\mathrm{A}, \mathrm{B}$, and $\mathrm{C}$ phases correspond to the presence of a magnetic plateau at $m=1 / 5,3 / 5$, and both $1 / 5$ and $3 / 5$, respectively. At the bottom of Fig. 2 we show a magnetization curve representative of each phase. Additionally, to evaluate the evolution of the plateaus along the phase diagram, we determined the plateau widths for each pair $\left(J_{3}, J_{1}\right)$. The $m=$ $1 / 5(m=3 / 5)$ plateau widths correspond to the top middle (right) diagram in Fig. 2.

\section{A. Correlation functions}

To study the magnetic order associated with the semiclassical plateaus of Fig. 2, we computed the $\left\langle S_{1}^{z} S_{n}^{z}\right\rangle$ correlation function, at $T=0$, using DMRG.

In Fig. 3 we present the results obtained for the correlation function vs $n$ (according to the numbering indicated in Fig. 1). From the top, the first (second) panel corresponds to the magnetic plateau of Fig. 2 bottom left (middle). The third and fourth panels of Fig. 3 correspond to the $m=1 / 5$ and $3 / 5$ magnetic plateaus of Fig. 2 bottom right, respectively.

The most important aspect to recall is that the correlation structure for all four plateaus analyzed here is in correspondence with those obtained in the Ising limit, although
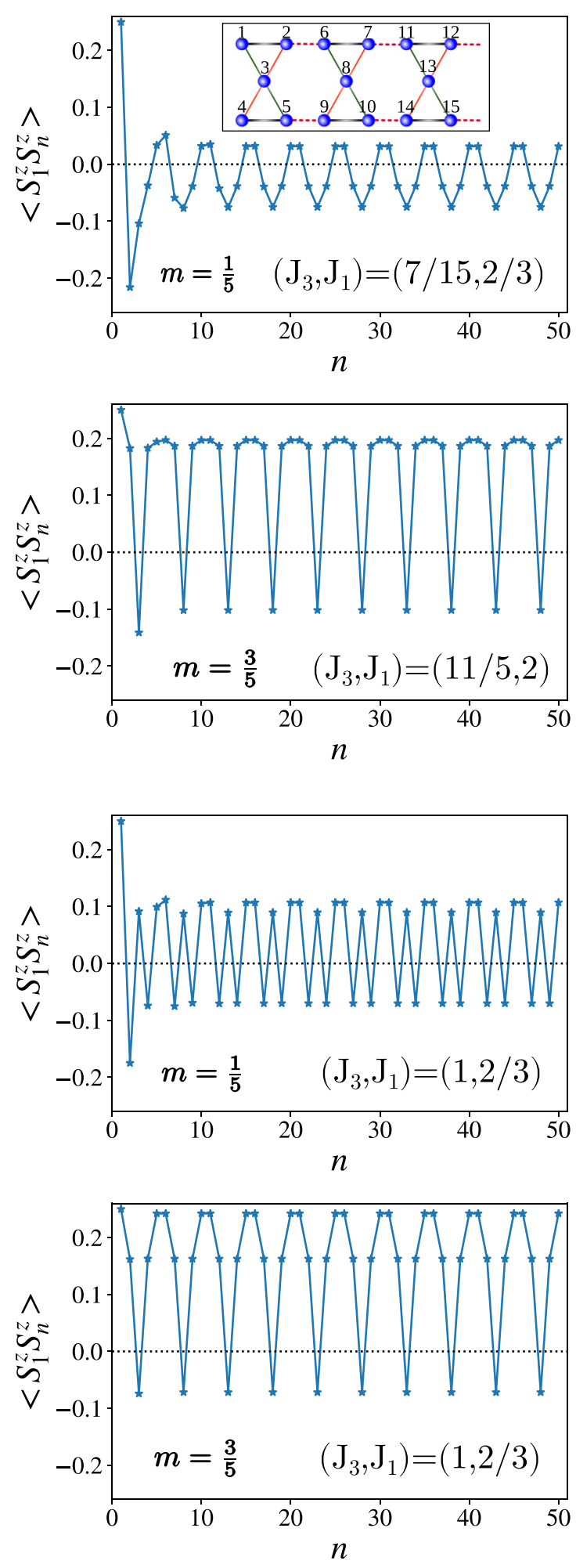

FIG. 3. $\left\langle S_{1}^{z} S_{n}^{z}\right\rangle$ correlation function on the magnetization plateaus representative from each phase shown in Fig. 2 (bottom), calculated by DMRG at $T=0$, with $J_{2}=1$. The index $n$ follows the indexation from the inset in the first panel of this figure, and $m$ denotes the plateau magnetization. Note that quantum fluctuations reduce the correlation amplitudes. However, the signature in all four plateaus is in complete agreement with the classical Ising limit, as we show in Secs. II C and II D. 

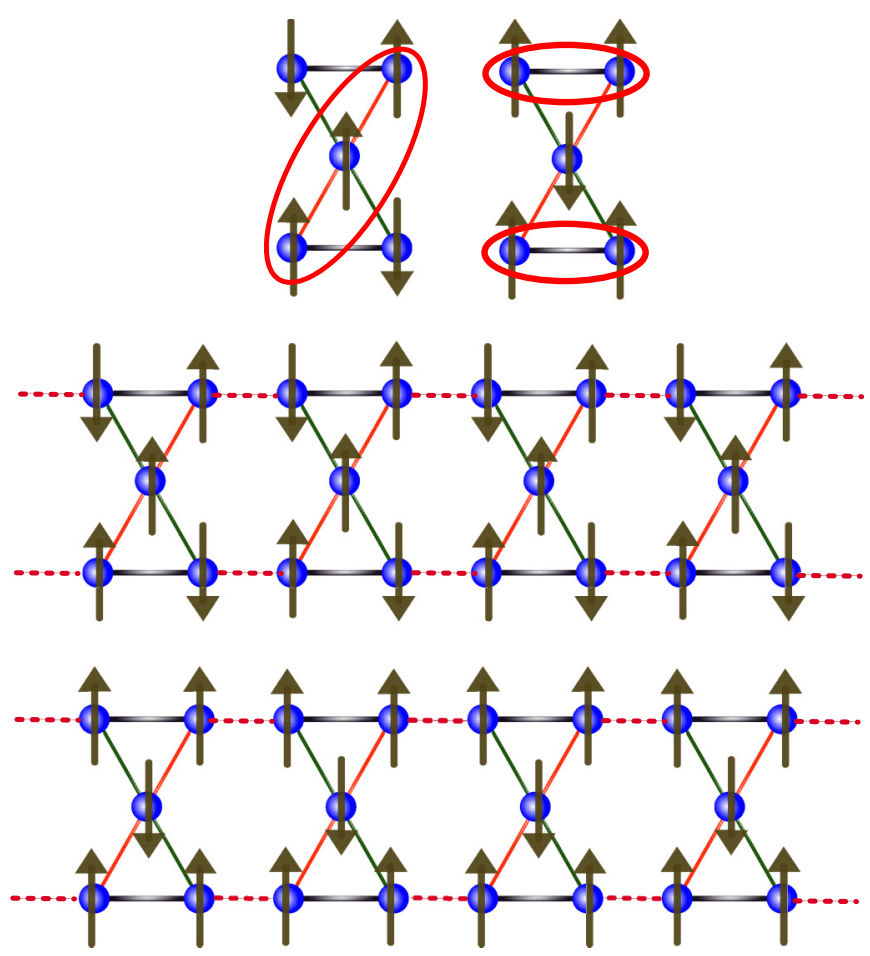

FIG. 4. Top left (right): Plaquette Ising ground state for $J_{2}>$ $J_{1}>J_{3}\left(J_{2}<\left\{J_{1}, J_{3}\right\}\right)$ with magnetization $m=1 / 5(m=3 / 5)$. Middle (bottom): Extension of local structures depicted above for finite $J_{4}>0$ in the kagome stripe with magnetization $m=1 / 5(m=3 / 5)$.

renormalized by quantum fluctuations. In the following subsections (Secs. IIC and IID) we explore this connection in detail from the perspective of a classical Ising model on the kagome stripe and from the isolated plaquettes, respectively.

\section{B. Ising limit of the kagome stripe}

In order to describe from a classical perspective the magnetic phases analyzed before, let us first consider the Ising limit of the isolated plaquette, i.e., $\vec{S}_{j}=\left(0,0, S_{j}^{z}\right)$ and $J_{4}=0$ in (1). The Hamiltonian for the plaquette in this case reads

$$
\begin{aligned}
H_{j}= & J_{2}\left(S_{j, 1}^{z} S_{j, 2}^{z}+S_{j, 4}^{z} S_{j, 5}^{z}\right)+J_{1} S_{j, 3}^{z}\left(S_{j, 1}^{z}+S_{j, 5}^{z}\right) \\
& +J_{3} S_{j, 3}^{z}\left(S_{j, 2}^{z}+S_{j, 4}^{z}\right) .
\end{aligned}
$$

It is possible to identify collinear ground states corresponding to Hamiltonian (3). Let us consider two different cases (both are two-degenerate due to spin inversion symmetry), depending of the coupling's ratio.

(1) Case I, $J_{2}>J_{3}>J_{1} \quad\left(J_{2}>J_{1}>J_{3}\right)$ : In this case $J_{1}\left(J_{3}\right)$ is frustrated and the magnetization of the plaquette is $m=1 / 5$. The last case is represented in Fig. 4 (top left).

(2) Case II, $J_{2}<\left\{J_{1}, J_{3}\right\}$ : In this case $J_{2}$ is frustrated (independently of the relative values of $J_{1}$ and $J_{3}$ ) and the magnetization of the plaquette is $m=3 / 5$, as represented in Fig. 4 (top right).

These local magnetic structures can be extended to the complete kagome-stripe lattice, where the individual plaquettes are coupled by $J_{4}>0$. For case I, the result of this interaction is a product state of $N$ individual plaquettes in

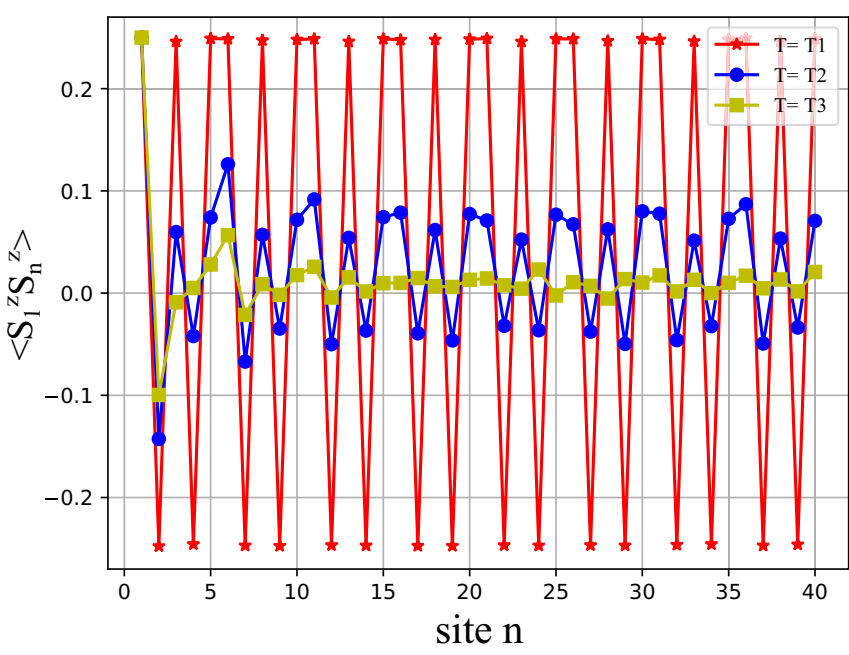

FIG. 5. Classical correlation function $\left\langle S_{1}^{z} S_{n}^{z}\right\rangle$ on the $m=1 / 5$ magnetization plateau $(h=1.0)$ calculated using a Monte Carlo simulation in the Ising limit of the model for temperatures $T_{1}=0.5$ (red), $T_{2}=1.1$ (blue), and $T_{3}=1.7$ (yellow), for $J_{1}=\frac{2}{3}, J_{2}=1$, and $J_{3}=1$ (same coupling constants as the third panel from the top of Fig. 3). For $T=T_{1}$ no thermal excitations are found in the system, indicating that the spins are parallel or antiparallel with each other. For $T=T_{3}$ the temperature is high enough for the correlations to rapidly fall off with $n$. For $T=T_{2}$ the correlations $\left\langle S^{z} S^{z}\right\rangle$ obtained by the classical Monte Carlo simulation are very similar to those calculated by DMRG using the Heisenberg model at $T=0$.

exactly the same state, and the (normalized) magnetization is still $m=1 / 5$, as shown in Fig. 4 (middle).

In case II at $h=0, J_{4}>0$ couples the plaquettes and the stripe has $m=0$. However, at high $h$, again we can construct a state with $N$ individual plaquettes in the same state, with $m=3 / 5$, as we depict in Fig. 4 (bottom).

The above shows that it is possible to construct classical states for the kagome stripe (Fig. 4) with the same magnetic structure of plateaus at $m=1 / 5$ and $3 / 5$, obtained via a fully quantum treatment of the model by means of DMRG (Fig. 2), which are consistent with Fig. 2 of Morita et al. [27]. In addition, the correlations calculated by DMRG (3) also show the same structure as the Ising case as it is further investigated in the following subsection.

\section{Quantum vs thermal fluctuations}

To compare the role of thermal fluctuations at classical level with zero-temperature quantum effects, we analyzed the finite temperature classical limit of the model. For this we carried out Monte Carlo simulations of the Ising model, i.e., $\vec{S}_{i}=\left(0,0, S_{i}^{z}\right)$, in (1), with the Metropolis algorithm [30], employing 500 sites and 1500 independent systems. To prevent the system from stopping in a local-energy minimum at low $T$, we performed an annealing process, starting with a high-temperature state (the system is in the paramagnetic phase) and then lowering the temperature progressively until no thermal fluctuations are found.

In Fig. 5 we present the results of the calculated correlations $\left\langle S_{1}^{Z} S_{n}^{z}\right\rangle$ for $T_{1}=0.5, T_{2}=1.1$, and $T_{3}=1.7$, with $T$ in units of energy. Note that for $T=T_{1}$ no thermal excitations 


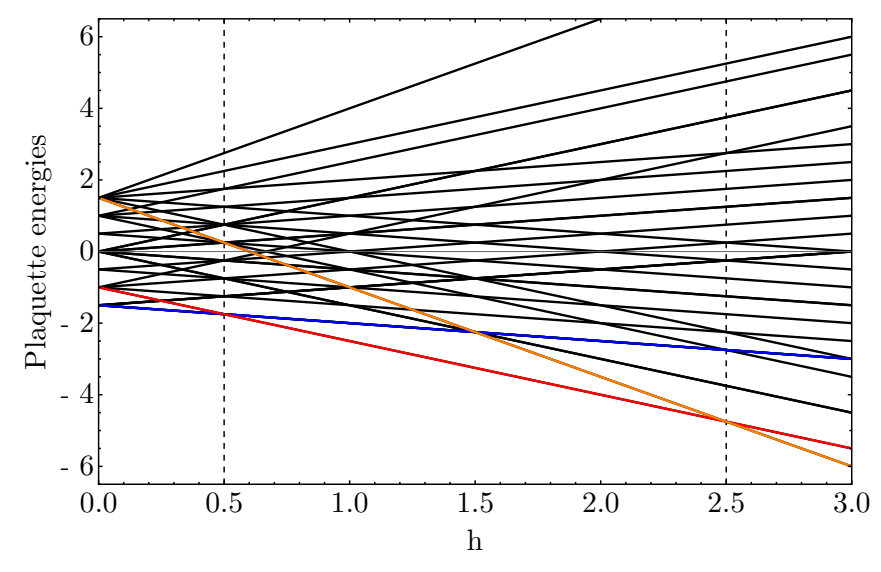

FIG. 6. Energies as functions of magnetic field for the isolated plaquette, for $J_{1}=J_{2}=J_{3}=1$. Ground states with magnetizations $m=1 / 5,3 / 5$, and 1 are indicated with blue, red, and orange lines, respectively. Critical magnetic fields $h_{0}^{(1)}=0.5$ and $h_{0}^{(2)}=2.5$ are highlighted by dashed vertical lines, where the ground state becomes three- and twofold degenerate, respectively.

are found in the system, indicating that the spins are parallel or antiparallel with each other. On the other hand, for $T=$ $T_{3}$ the temperature is high enough for the correlations to rapidly fall off with $n$. Finally, for the intermediate $T=T_{2}$ we highlight that the correlations $\left\langle S_{1}^{z} S_{n}^{z}\right\rangle$ obtained by the classical Monte Carlo simulation are very similar to those calculated by DMRG using the Heisenberg model at $T=0$. This indicates that the effect of quantum and thermal fluctuations gives an analogous result in this correlation function.

\section{Effective model on semiclassical plateaus}

Here we present an analytical approach for the treatment of semiclassical plateaus, which complements the numerical methods considered before. The method consists in the construction of an effective Hamiltonian, based in quantum degenerate perturbation theory [31-36]. In the last part of the paper we apply the effective model technique for the case of quantum plateaus that break the translational invariance of the lattice.

For the present case, we start by considering a system of isolated plaquettes, i.e., $J_{4}=0$ (see Fig. 1), the Hilbert space of which has dimension $d=2^{5}$. For fixed values of the couplings $J_{1}, J_{2}$, and $J_{3}$, it is possible to diagonalize numerically the plaquette Hamiltonian and obtain all the energies as functions of the magnetic field $h$. This is illustrated in Fig. 6 for the homogeneous plaquette case $\left(J_{1}=J_{2}=J_{3}=1\right)$.

Note that for $h>0$ the plaquette has three different ground states corresponding to magnetizations $m=1 / 5$ (blue), $m=$ $3 / 5$ (red), and $m=1$ (orange), depending on the magnetic field value. At the critical fields $h_{0}^{(1)}$ and $h_{0}^{(2)}$ (corresponding to the dashed vertical lines in the figure), the ground state becomes degenerate. In particular, at $h_{0}^{(1)}$ the ground state is threefold degenerate, although this is a particularity of the homogeneous case where all the couplings are equal.

The next step is to consider a weak coupling between the plaquettes, in particular, at the level crossing.
For $J_{4}$ finite, we separate the complete Hamiltonian in two terms:

$$
H=H_{0}+H_{\text {int }},
$$

where

$$
\begin{aligned}
H_{0}= & \sum_{n}\left[J_{1}\left(\vec{S}_{n, 1} \cdot \vec{S}_{n, 3}+\vec{S}_{n, 3} \cdot \vec{S}_{n, 5}\right)\right. \\
& +J_{2}\left(\vec{S}_{n, 1} \cdot \vec{S}_{n, 2}+\vec{S}_{n, 4} \cdot \vec{S}_{n, 5}\right) \\
& \left.+J_{3}\left(\vec{S}_{n, 2} \cdot \vec{S}_{n, 3}+\vec{S}_{n, 3} \cdot \vec{S}_{n, 4}\right)-h_{0} \sum_{m=1}^{5} S_{n, m}^{z}\right]
\end{aligned}
$$

corresponds to the Hamiltonian of a single plaquette, where $h_{0}$ is the magnetic field at the energy-level crossing, and

$$
\begin{aligned}
H_{\mathrm{int}}= & \sum_{n}\left[J_{4}\left(\vec{S}_{n, 2} \cdot \vec{S}_{n+1,1}+\vec{S}_{n, 5} \cdot \vec{S}_{n+1,4}\right)\right. \\
& \left.-\left(h-h_{0}\right) \sum_{m=1}^{5} S_{n, m}^{z}\right]
\end{aligned}
$$

is the plaquettes-interaction term.

Considering $0<J_{4}, h-h_{0} \ll J_{i}, i=1,2,3$, at first order of perturbation theory we have

$$
H^{(1)}=\sum_{i j}\left|p_{i}\right\rangle\left\langle p_{i}\left|H_{\mathrm{int}}\right| p_{j}\right\rangle\left\langle p_{j}\right|,
$$

where $\left|p_{i}\right\rangle$ are the $2^{N_{c}}$ degenerated ground states, $N_{c}$ being the number of unit-cell plaquettes. Computing (7) and expanding the result locally in the $\left\{\sigma^{0}, \frac{\sigma^{i}}{2}\right\}$ basis, where $\sigma^{0}$ is the $2 \times 2$ identity matrix and $\sigma^{i}$ are the Pauli matrices, one arrives (up to a constant term) at a low-energy effective Hamiltonian corresponding to a spin-1/2 anisotropic Heisenberg chain with only nearest-neighbor interactions:

$$
H_{\mathrm{eff}}=\sum_{n} J_{x y}\left(S_{n}^{x} S_{n}^{x}+S_{n}^{y} S_{n}^{y}\right)+J_{z z} S_{n}^{z} S_{n}^{z}-\tilde{h} S_{n}^{z},
$$

in which the effective couplings $J_{x y}$ and $J_{z z}$ and the effective magnetic field $\tilde{h}$ depend on the original couplings $J_{i}$ and magnetic field $h$. Note that this model is valid for twofold degenerate local ground states, which translates into an effective spin-1/2 per site.

For large enough $\tilde{h}$, the ground state of (8) is the magnon vacuum $|0\rangle \equiv|\uparrow \uparrow \uparrow \uparrow \ldots\rangle$ (or $|\downarrow \downarrow \downarrow \downarrow \ldots\rangle)$.

We now compute the one-magnon dispersion relation:

$$
\epsilon_{ \pm}(k)=J_{x y} \cos (k)-J_{z z} \pm \tilde{h} .
$$

From (9) we calculate the edges of the plateaus around the critical field where the first-order expansion is made. We impose the condition of gap closure, which determines the edge of the plateaus in terms of the magnetic couplings of the effective model:

$$
\tilde{h}= \pm\left(J_{x y}+J_{z z}\right)
$$

In Fig. 7 we depict four sectors of magnetization curves showing the transition between $m=3 / 5$ and 1 plateaus. The green dashed lines mark the plateau edges as calculated by the low-energy effective Hamiltonian technique, using (10), for 

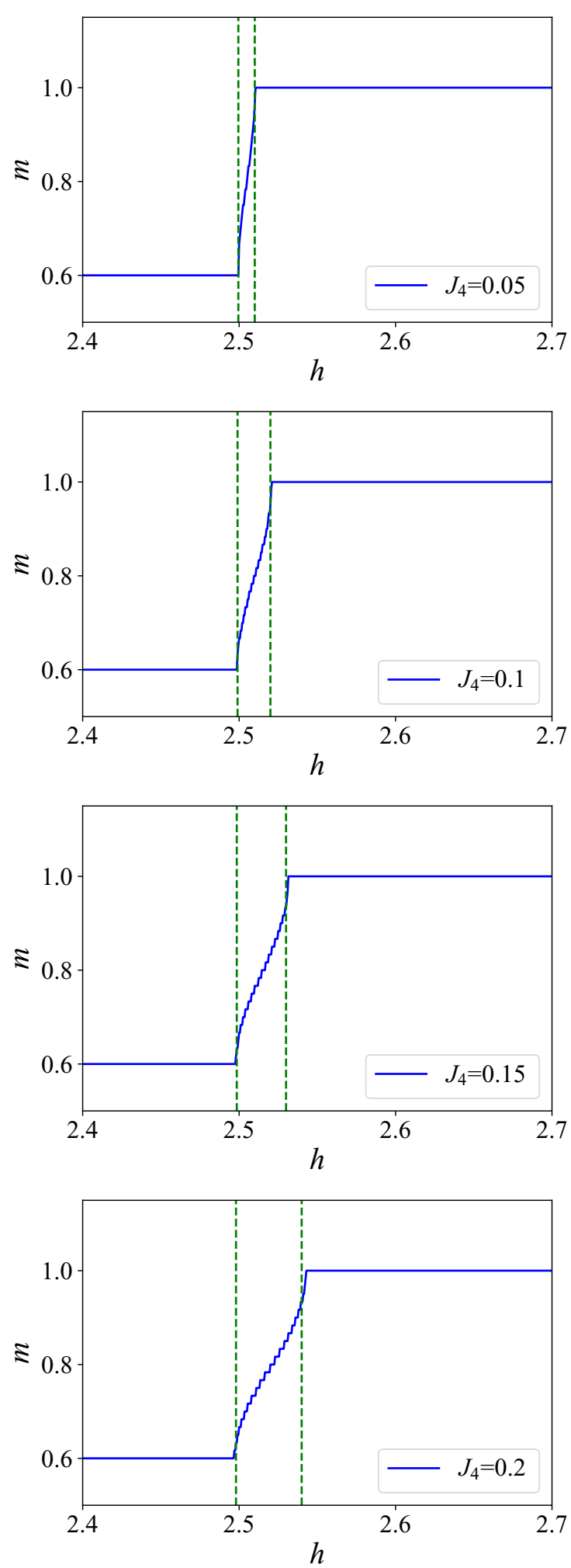

FIG. 7. Magnetization curves near saturation for $J_{i}=1, i=$ $1,2,3$ and $J_{4}=0.05,0.1,0.15,0.2$ from top to bottom, respectively. Green dashed lines correspond to the edge of the plateaus predicted by the closure of the gap in the magnon dispersion (9).

$J_{4}=0.05,0.1,0.15,0.2$ and $J_{i}=1, i=1,2,3$. We found a very good agreement in the perturbative regime between analytical and numerical results.

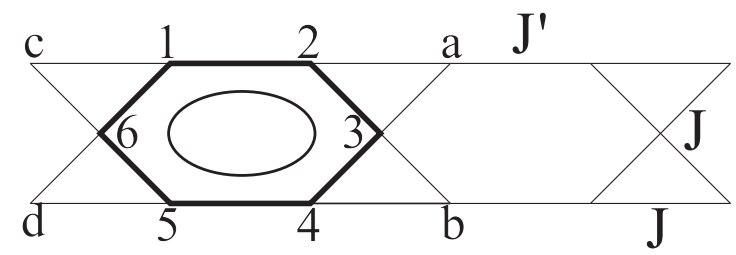

FIG. 8. Kagome-stripe scheme where the bold hexagon denotes the region $L$, and the ellipse represents a localized magnon. With numbers we index the sites of $L$, and with letters $a, b, c$, and $d$ we index the sites in interaction with the region $L$. Here we show only two couplings, $J^{\prime}$ and $J$, both subject to (13).

The treatment for the case $m=1 / 5$ is conceptually similar to $m=3 / 5$ and it has also been studied numerically in other works [27]; we will not elaborate more on the subject here.

This concludes our identification of classical plateaus. In Sec. III B the presence of quantum plateaus is studied. These plateaus will not have a classical counterpart correspondence but a purely quantum-mechanical origin which breaks the lattice symmetry.

\section{QUANTUM PLATEAUS OF LOCALIZED MAGNONS}

In this section we study phases with spontaneous breaking of the lattice translational symmetry, in which frustration induces a periodic structure of localized magnons associated to intermediate (or fractional) plateaus. Noteworthy, we find an exact magnon crystal ground state with $m=4 / 5$ of the anisotropic Heisenberg model, which is a generalization of the state reported by Schulenburg et al. [21].

\section{A. Magnon crystal phase in the anisotropic kagome stripe}

Let us first consider the anisotropic version of the Heisenberg model on the kagome stripe (1) in a magnetic field, the Hamiltonian of which reads

$$
H=\sum_{\langle i, j\rangle} J_{i j}\left[\Delta S_{i}^{z} S_{j}^{z}+\frac{1}{2}\left(S_{i}^{+} S_{j}^{-}+S_{i}^{-} S_{j}^{+}\right)\right]-h S^{z} .
$$

At high magnetic field the ground state is the fully polarized ferromagnetic state $|0\rangle \equiv|\uparrow \uparrow \uparrow \uparrow \ldots\rangle$ and the lowest-energy excitations can be written in terms of a linear combination of one-magnon states as

$$
|1\rangle=\sum_{l} a_{l} S_{l}^{-}|0\rangle .
$$

Taking a particular set of coupling values, the magnon dispersion relation may be independent of the momentum $k$, giving rise to a flat band spectrum. This implies that magnon excitations can be localized in a finite region of the stripe.

It is possible to construct the exact eigenstate of (11), with localized magnons in the region $L$ represented by the bold hexagon in Fig. 8.

The necessary and sufficient condition for decoupling of the local state from the rest of the system is

$$
\sum_{l \in L} a_{l} J_{l \alpha}=0,
$$


where $J_{l \alpha}$ couples the spins $\vec{S}_{l}$ and $\vec{S}_{\alpha}$, with $l \epsilon L$ and $\alpha=$ $a, b, c, d$ (see Fig. 8). It is possible to satisfy (13) by taking

$$
a_{l}=\frac{(-1)^{l}}{\sqrt{6}}, l \epsilon L,
$$

with the indexation of Fig. 8, and the coupling condition

$$
J^{\prime}=J \frac{2 \Delta+1}{\Delta+1}
$$

where $J^{\prime}$ couples contiguous two-triangle-cells (the unit cell framed in Fig. 1) and $J$ couples spins inside this unit cell as shown in Fig. 8. This is the exact state reported by Schulenburg et al. [21].

The exact ground state of (11) previous to saturation is the state with $N / 2$ localized noninteracting magnons (with $N$ the number of unit cells) and presents magnetization $m=$ $4 / 5$. The presence of this exact solution can be observed in the magnetization curve as a jump of $\delta m=1 / 5$ just below saturation [21]. Moreover, a fully exactly factorized ground state has necessary short-range entanglement entropy. Reciprocally, the property of gapped systems to present short-range entanglement entropy [37] gives further indications that, at this point, the system is fully gapped, implying, in particular, the presence of a magnetization plateau. For example, imagine that one wishes to describe the low-energy behavior of the system in this point with a field theory description. Among the degrees of freedom of the low-energy description, the magnetic sector is the one related to the presence of a plateau in the magnetization curve: a gap in this sector implies a plateau. On the other hand, in order to obtain a short-range entanglement, this field theory should contain only shortranged or gapped degrees of freedom. This then implies a gap also in the magnetic sector and thus the presence of a magnetization plateau.

We have constructed the magnetization curve of model (11) satisfying the coupling condition (15) for several values of anisotropy $\Delta$, by means of DMRG. The results are shown in Fig. 9, where a macroscopic magnetization jump to saturation can be observed. In addition, a magnetization plateau is present at $m=4 / 5$. This plateau is consistent with the OYA theorem 2 provided that the ground state unit cell contains ten spins. Therefore, the system breaks spontaneously the original lattice translation symmetry, doubling the size of the unit cell as is expected for the noninteracting localized magnon state.

\section{B. Effective model on quantum plateaus of localized magnons}

Here we further study the nature of plateaus with spontaneous breaking of the translational symmetry, in particular $m=4 / 5$ and the connection with localized magnons. To this end we constructed a low-energy effective Hamiltonian via degenerate perturbation theory. First note that, according to (15), $J^{\prime}(\Delta=1)=3 / 2 J$. This suggests the use of a more convenient unit cell including the strongest coupling $J^{\prime}$ as shown in Fig. 10. The unit cells (which we call "pencil cells") contain two different couplings $J$ and $K$ and are interconnected via $J_{2}$ and $K_{2}$.

To illustrate the method we fixed $(J, K)=(1.5,1)$, so that for $\left(J_{2}, K_{2}\right)=(1,1)$ the model satisfies $(15)$ and the system is

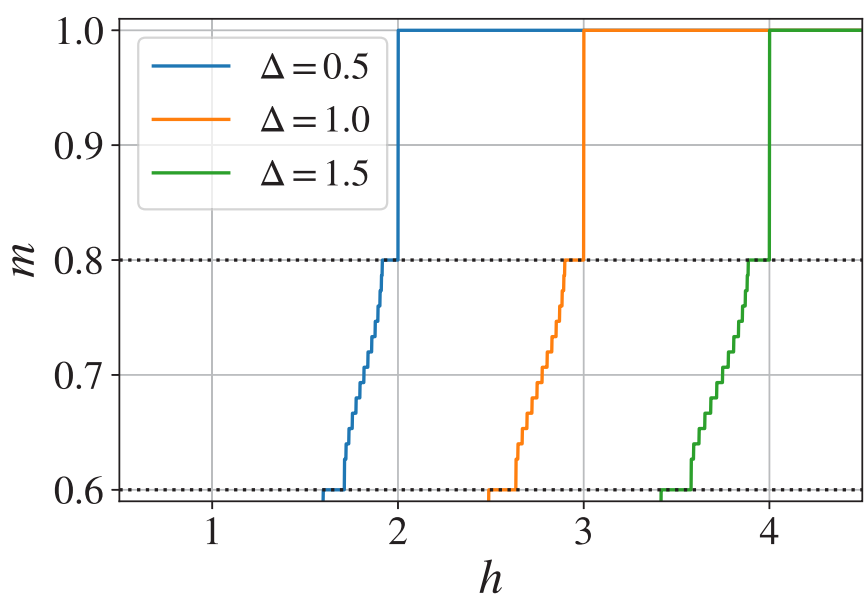

FIG. 9. Macroscopic magnetization jump $(\delta m=1 / 5)$ to saturation calculated by DMRG for a strip with 150 spins, on the coupling condition (15) for three different anisotropies, which shows numerically the result of the presence of $N / 2$ noninteracting magnons in the stripe as a first excited state. Additionally, there is a $m=4 / 5$ plateau.

in an exact magnon crystal state, which we call from now on "point I."

Our effective model starts from decoupled pencil cells, i.e., $\left(J_{2}, K_{2}\right)=(0,0)$, rendering point I difficult to access perturbatively. However, we will see that the model captures properly the phases with $m=4 / 5$, associated with localized magnons.

To construct the effective model we start by separating the Hamiltonian into $H=H_{0}+H_{\text {int }}$, where

$$
\begin{aligned}
H_{0}= & \sum_{n}\left[J\left(\vec{S}_{n, 1} \cdot \vec{S}_{n, 2}+\vec{S}_{n, 5} \cdot \vec{S}_{n, 4}\right)\right. \\
& \left.+K\left(\vec{S}_{n, 2} \cdot \vec{S}_{n, 3}+\vec{S}_{n, 3} \cdot \vec{S}_{n, 4}\right)-h_{0} \sum_{m=1}^{5} S_{n, m}^{z}\right],
\end{aligned}
$$

in which $h_{0}$ is, as in Sec. IID, the magnetic field where the isolated pencil-plaquette ground state becomes degenerated due to the level crossing, and

$$
\begin{aligned}
H_{\mathrm{int}}= & \sum_{n}\left[J_{2}\left(\vec{S}_{n, 2} \cdot \vec{S}_{n+1,1}+\vec{S}_{n, 4} \cdot \vec{S}_{n+1,5}\right)\right. \\
& \left.+K_{2}\left(\vec{S}_{n, 3} \cdot \vec{S}_{n+1,1}+\vec{S}_{n, 3} \cdot \vec{S}_{n+1,5}\right)-\left(h-h_{0}\right) \sum_{m=1}^{5} S_{n, m}^{z}\right] .
\end{aligned}
$$

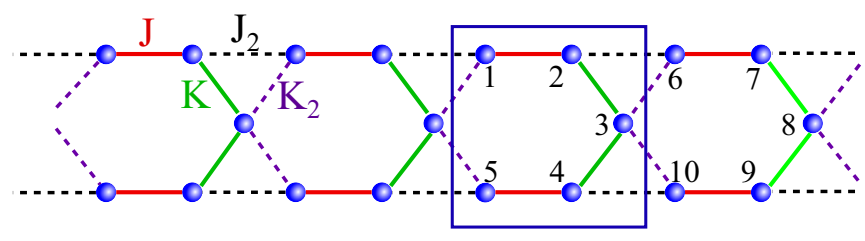

FIG. 10. Kagome-stripe scheme using the "pencil unit cell" (framed with blue lines). This unit cell is not frustrated and composed by five sites, with two couplings, $J$ and $K$, fixed here to 1.5 and 1 , respectively. The cells are coupled by $J_{2}$ and $K_{2}$. The numbers indicate the site indexation. 


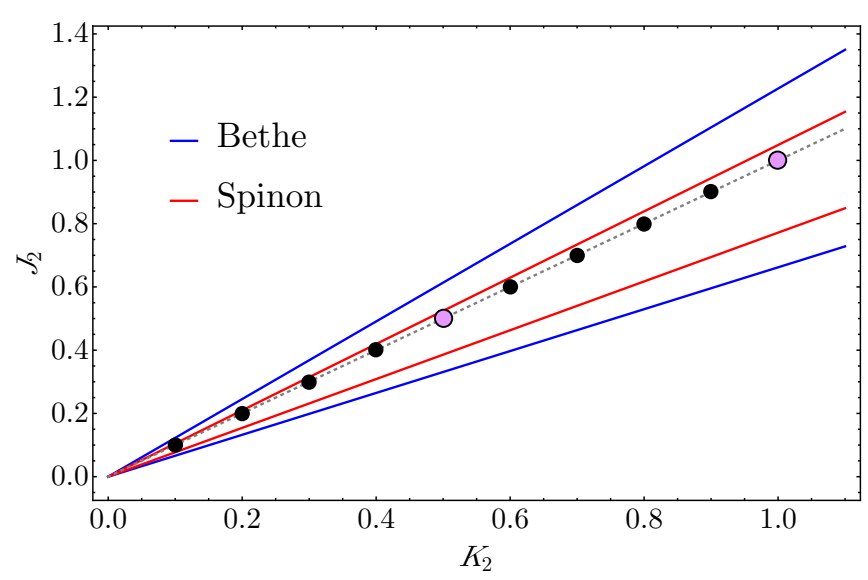

FIG. 11. Blue lines: The limits of the region where the effective Hamiltonian predicts the presence of a fractional $m=4 / 5$ plateau using the Bethe ansatz. Red lines: The corresponding result using the spinon dispersion relation approach on the effective model. The solid circles indicate the points selected to evaluate, by means of DMRG, the magnetization curves (Fig. 12) and magnon localization (Fig. 13). In particular, pink circles denote the presence of an exact solution, the crystal magnon phase, of localized magnons.

By performing first-order perturbation theory as before, we get a low-energy effective Hamiltonian that predicts a region in couplings space where a fractional plateau at $m=4 / 5$ emerges. We proceeded in two ways, as shown in Fig. 11. In blue we plot the solutions for $\Delta=1$ in the effective model which, according to the Bethe ansatz [38], indicates that the effective chain does not pass through the Néel phase [36], and consequently the kagome stripe does not have a fractional plateau.

In red, we plot the solutions for a gapless spinon dispersion relation over the Néel state where both critical magnetic fields

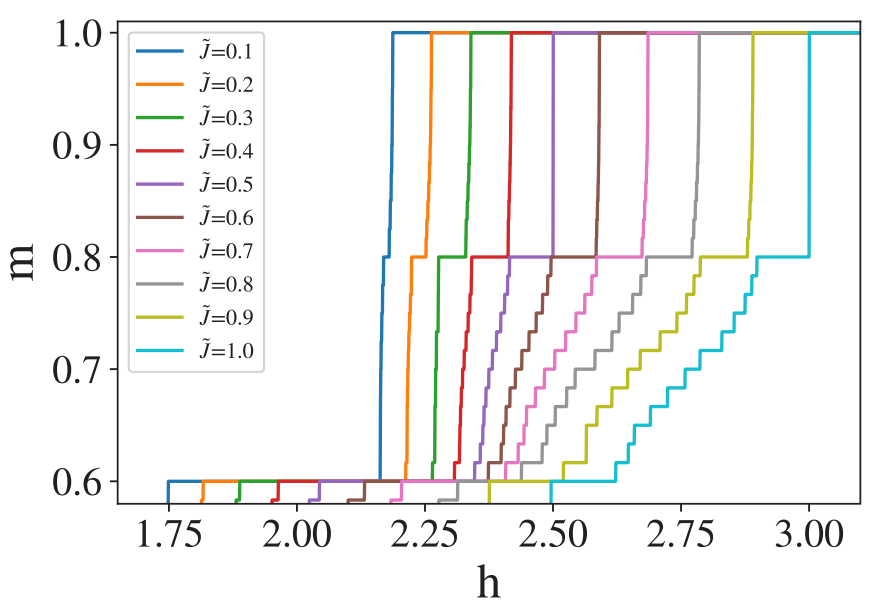

FIG. 12. Partial magnetization curves computed by means of DMRG for $J=3 / 2, K=1$ and $J_{2}=K_{2} \equiv \tilde{J}=0.1,0.2, \ldots, 1$ using 24 pencil unit cells. Although there is a large slope transition in all the cases, for $\tilde{J}=0.5$ there is a clean jump from $m=4 / 5$ to saturation, as in the $\tilde{J}=1$ case (point I). This is a signature of a second exact solution of localized magnons which we named "point II."
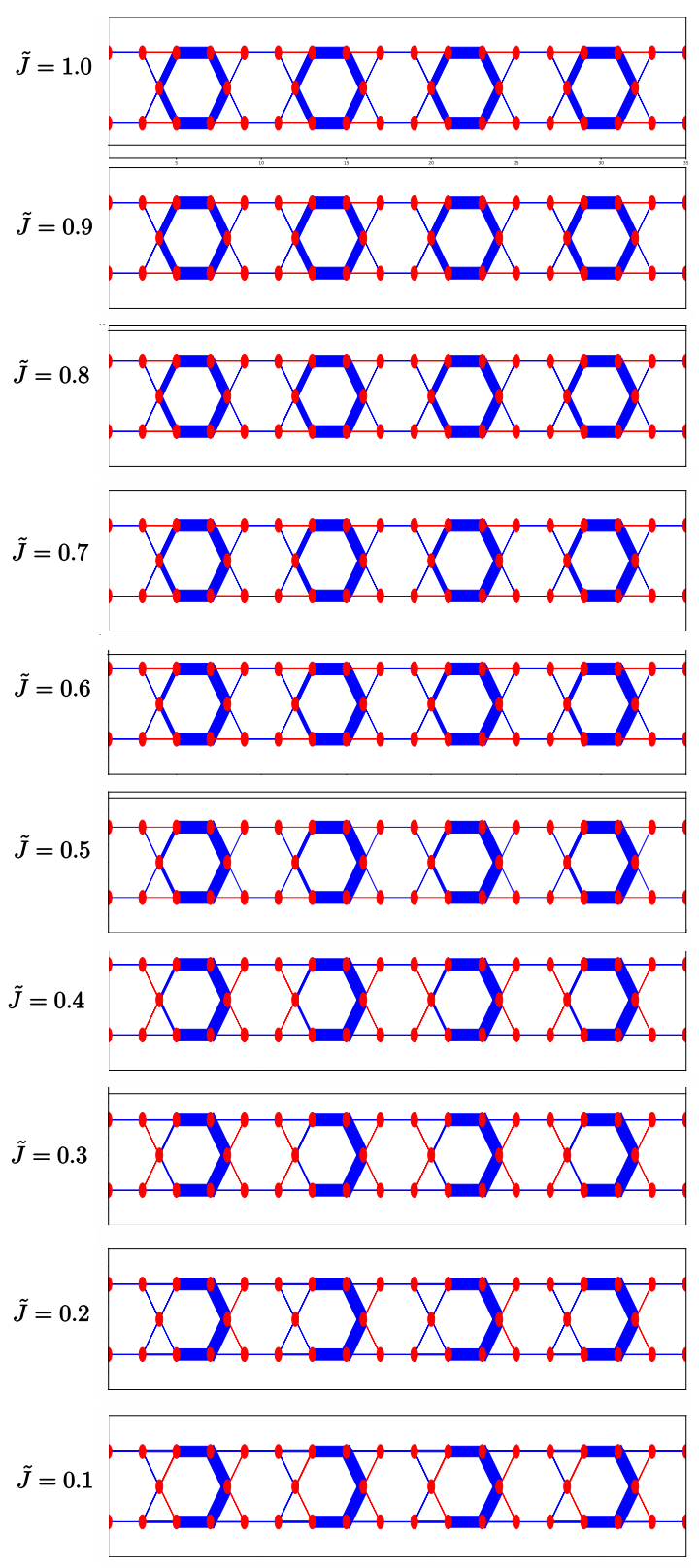

FIG. 13. Transition between hexagon and pencil cell localized magnons. Linewidths indicate the $\left\langle S_{i}^{+} S_{j}^{-}\right\rangle$correlation, for $\Delta=1$, $J=3 / 2, K=1$ and $K_{2}=J_{2} \equiv \tilde{J}=0.1,0.2, \ldots, 1$, computed by DMRG. Blue (red) lines correspond to negative (positive) correlations. The fluctuations are located on hexagons or pencil cells for $\tilde{J}$ near the unity or zero, respectively.

(plateau edges) are equal. Both regions contain the exact solution $\left(J_{2}, K_{2}\right)=(1,1)$ (point $\mathrm{I}$ ), represented by a pink circle in Fig. 11, although the model is truly valid only in the $J_{2}, K_{2} \ll J, K$ limit.

To analyze the evolution of the fractional $m=4 / 5$ plateau from the perturbative regime until reaching point I, we evaluated numerically the magnetization curves by DMRG in the line $J_{2}=K_{2} \equiv \tilde{J}$, for $\tilde{J}=0.1,0.2, \ldots, 1$ (points represented by circles in Fig. 11), with $\Delta=1$.

The results are presented in Fig. 12. As it can be observed in all cases there is a transition to saturation with a large slope, 


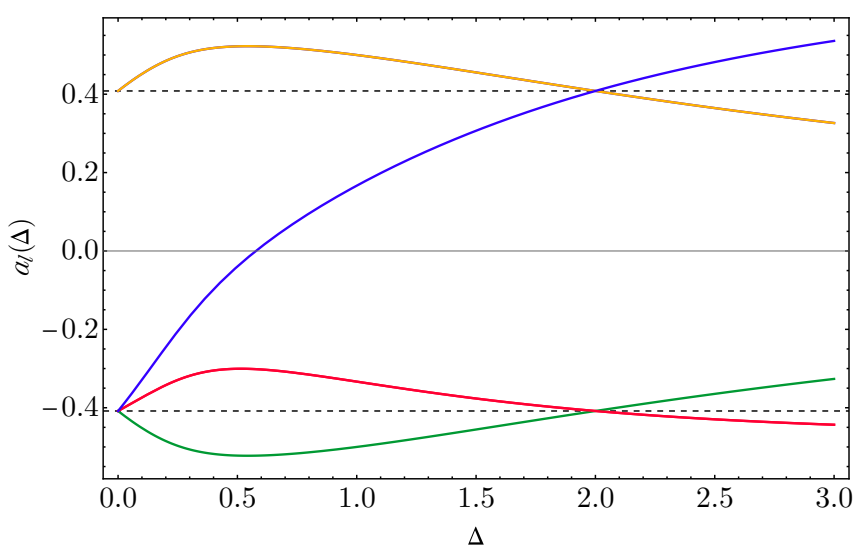

FIG. 14. Amplitudes $a_{1}=a_{5}$ (pink), $a_{2}=a_{4}$ (yellow), $a_{3}$ (green), and $a_{6}$ (violet) as functions of the anisotropy $\Delta$, for the generalization of the magnon crystal phase of point II. Dashed horizontal lines show the solution (14) (exact ground state in point I). Both states are equal at $\Delta=2$.

together with an increase in the $m=4 / 5$ plateau width. In particular, for $\tilde{J}=0.5$ there is an abrupt jump of $\delta m=1 / 5$, identical to the magnetization jump observed for point I $(\tilde{J}=$ 1). Motivated by this result, we proposed a state of the form (12) and found a second exact solution of localized magnons, that we named "point II," represented by a pink circle at $J_{2}=$ $K_{2}=0.5$ in Fig. 11.

To analyze the transition between hexagon and pencil cell localized magnons, we numerically evaluated the $\left\langle S_{i}^{+} S_{j}^{-}\right\rangle$ correlation functions, by means of DMRG. The results are presented in Fig. 13, where we show $\left\langle S_{i}^{+} S_{j}^{-}\right\rangle$for $J_{2}=K_{2} \equiv$ $\tilde{J}=0.1,0.2, \ldots, 1$ (corresponding to the ten dots in Fig. 11). As it can observed, in the homogeneous case where $\tilde{J}=1$ the fluctuations are localized on the hexagons as predicted. Note that this particular case is also depicted in Fig. 5(a) of Morita et al. [27]. On the other hand, as $\tilde{J}$ decreases, the fluctuations localize on pencil cells progressively.

\section{Generalized magnon crystal phase}

To generalize the solution in point II to the anisotropic case $(\Delta \neq 1)$ we proposed a coupling set $\left\{J=\frac{2 \Delta+1}{\Delta+1} K, K=\right.$ $\left.1, J_{2}(\Delta)=K_{2}(\Delta)\right\}$ (following the notation from Fig. 10) and we found the solution

$$
\frac{K_{2}(\Delta)}{K}=\frac{\sqrt{12 \Delta^{3}(\Delta+1)+1}-(2 \Delta+1)}{2 \Delta(\Delta+1)},
$$

together with a set of couplings $a_{l}(\Delta), l \epsilon L$, plotted in Fig. 14 .

We highlight that for $\Delta=2$ the coefficients $a_{l}$ are exactly as in (14), as shown in Fig. 14; while the couplings are $\left\{J=5 / 3, K=1, J_{2}=K_{2}=1\right\}$. In addition, $K_{2}(\Delta=2)$ and
$K$ have the same value as in the point-I case, while $J$ is different. Finally let us note that, for $\Delta \epsilon(0,1 / \sqrt{3}),(18)$ is negative, indicating ferromagnetic couplings.

\section{CONCLUSIONS}

In the present paper we studied the magnetization properties of an antiferromagnetic kagome-stripe lattice. We constructed a magnetic phase diagram which shows three magnetic phases with the presence of $m=1 / 5$ or $3 / 5$ magnetic plateaus, or both simultaneously. These plateaus are classical in the sense that they can be understood in terms of the Ising limit, by studying the magnetization curves and the $\left\langle S_{i}^{z} S_{j}^{z}\right\rangle$ correlation function as well as comparing quantum DMRG calculations with classical Monte Carlo simulations in the Ising ground state for different coupling configurations.

We calculated the plateau edges by means of the lowenergy effective Hamiltonian technique in the strong plaquettes limit. The same technique proved to be remarkably useful in predicting the presence of a fractional $m=4 / 5$ quantum plateau, a plateau that cannot be explained in the Ising Limit. This plateau is bounded to the presence of a localized magnon phase, as can be seen by computing the $\left\langle S_{i}^{+} S_{j}^{-}\right\rangle$correlation function with DMRG. Furthermore, we found an exact ground state with $m=4 / 5$ (just before saturation, due to a magnetization jump) of the anisotropic Heisenberg Hamiltonian, that provides a generalization of the state found by Schulenburg et al. [21]. This gives another example of an exactly factorized magnon crystal ground state which finds its origin in the strong frustrating nature of the Hamiltonian [39,40].

From a more general point of view, magnon crystals are known to be present in a wide variety of one- and twodimensional frustrated systems [21]. The hallmark of these system is a magnon flat band producing an exactly factorized ground state of localized magnons which is purely quantum mechanical. The magnetic phase diagram of the model studied here has the richness of having both this kind of factorized quantum state as well as magnetization classical (Ising like) plateaus. In particular, this phenomenology is also present in the fully two-dimensional kagome model, indicating that some essential aspects of the system transcend dimensionality. This has been an additional motivation to study the kagomestripe model, which also provides a more accessible numerical treatment.

Finally, the richness of this system makes it an ideal laboratory for studying the behavior of such different gapped states in the presence of perturbations like transverse field or Dzyaloshinskii-Moriya interactions.

\section{ACKNOWLEDGMENTS}

We acknowledge useful discussions with M. Matera. C.A.L. is supported by ANPCyT (PICT Grant No. 20130009).
[1] L. Balents, Nature (London) 464, 199 (2010).

[2] C. Lhuillier and G. Misguich, in Introduction to Frustrated Magnetism (Springer-Verlag, Berlin, 2011).

[3] L. Savary and L. Balents, Rep. Prog. Phys. 80, 016502 (2016).
[4] J. Knolle and R. Moessner, Annu. Rev. Condens. Matter Phys. 10, 451 (2019).

[5] J. Wen, S.-L. Yu, S. Li, W. Yu, and J.-X. Li, npj Quantum Mater. 4, 12 (2019). 
[6] M. P. Shores, E. A. Nytko, B. M. Bartlett, and D. G. Nocera, J. Am. Chem. Soc. 127, 13462 (2005).

[7] M. Zhesheng, H. Ruilin, and Z. Xiaoling, Acta Geol. Sin. (Engl. Ed.) 4, 145 (1991).

[8] F. H. Aidoudi, D. W. Aldous, R. J. Goff, A. M. Slawin, J. P. Attfield, R. E. Morris, and P. Lightfoot, Nat. Chem. 3, 801 (2011).

[9] M. Hermele, Y. Ran, P. A. Lee, and X.-G. Wen, Phys. Rev. B 77, 224413 (2008).

[10] S. Yan, D. A. Huse, and S. R. White, Science 332, 1173 (2011).

[11] T.-H. Han, J. S. Helton, S. Chu, D. G. Nocera, J. A. RodriguezRivera, C. Broholm, and Y. S. Lee, Nature (London) 492, 406 (2012).

[12] M. Fu, T. Imai, T.-H. Han, and Y. S. Lee, Science 350, 655 (2015).

[13] B. Bauer, B. P. Keller, S. Trebst, and A. W. W. Ludwig, Phys. Rev. B 99, 035155 (2019).

[14] D. W. Proukakis, N. P. Snoke and P. B. Littlewood, in Universal Themes of Bose-Einstein Condensation (Cambridge University, Cambridge, England, 2017).

[15] T. Giamarchi, C. Rüegg, and O. Tchernyshyov, Nat. Phys. 4, 198 (2008).

[16] V. Zapf, M. Jaime, and C. D. Batista, Rev. Mod. Phys. 86, 563 (2014).

[17] M. Takigawa and F. Mila, Magnetization Plateaus, Springer Series in Solid-State Sciences Vol. 164 (Springer, New York, 2011), Chap. 10, pp. 241-267.

[18] Y. Shirata, H. Tanaka, A. Matsuo, and K. Kindo, Phys. Rev. Lett. 108, 057205 (2012).

[19] H. Kageyama, K. Yoshimura, R. Stern, N. V. Mushnikov, K. Onizuka, M. Kato, K. Kosuge, C. P. Slichter, T. Goto, and Y. Ueda, Phys. Rev. Lett. 82, 3168 (1999).

[20] C. Gerhardt, K.-H. Mütter, and H. Kröger, Phys. Rev. B 57, 11504 (1998).

[21] J. Schulenburg, A. Honecker, J. Schnack, J. Richter, and H.-J. Schmidt, Phys. Rev. Lett. 88, 167207 (2002).

[22] M. E. Zhitomirsky and H. Tsunetsugu, Prog. Theor. Phys. Suppl. 160, 361 (2005).
[23] S. Nishimoto, N. Shibata, and C. Hotta, Nat. Commun. 4, 2287 (2013).

[24] S. Capponi, O. Derzhko, A. Honecker, A. M. Läuchli, and J. Richter, Phys. Rev. B 88, 144416 (2013).

[25] R. Okuma, D. Nakamura, T. Okubo, A. Miyake, A. Matsuo, K. Kindo, M. Tokunaga, N. Kawashima, S. Takeyama, and Z. Hiroi, Nat. Commun. 10, 1229 (2019).

[26] Y. Tang, W. Guo, H. Xiang, S. Zhang, M. Yang, M. Cui, N. Wang, and Z. He, Inorg. Chem. 55, 644 (2016).

[27] K. Morita, T. Sugimoto, S. Sota, and T. Tohyama, Phys. Rev. B 97, 014412 (2018).

[28] M. Oshikawa, M. Yamanaka, and I. Affleck, Phys. Rev. Lett. 78, 1984 (1997).

[29] B. Bauer, L. D. Carr, H. G. Evertz, A. Feiguin, J. Freire, S. Fuchs, L. Gamper, J. Gukelberger, E. Gull, S. Guertler, A. Hehn, R. Igarashi, S. V. Isakov, D. Koop, P. N. Ma, P. Mates, H. Matsuo, O. Parcollet, G. Pawłowski, J. D. Picon, L. Pollet, E. Santos, V. W. Scarola, U. Schollwöck, C. Silva, B. Surer, S. Todo, S. Trebst, M. Troyer, M. L. Wall, P. Werner, and S. Wessel, J. Stat. Mech. (2011) P05001.

[30] D. P. Landau and K. Binder, in A Guide to Monte Carlo Simulations in Statistical Physics (Springer-Verlag, Berlin, 2014).

[31] K. Totsuka, Phys. Rev. B 57, 3454 (1998).

[32] F. Mila, Eur. Phys. J. B 6, 201 (1998).

[33] K. Tandon, S. Lal, S. K. Pati, S. Ramasesha, and D. Sen, Phys. Rev. B 59, 396 (1999).

[34] F. Michaud, T. Coletta, S. R. Manmana, J.-D. Picon, and F. Mila, Phys. Rev. B 81, 014407 (2010).

[35] F. A. Gómez Albarracín, M. Arlego, and H. D. Rosales, Phys. Rev. B 90, 174403 (2014).

[36] F. Elias, M. Arlego, and C. A. Lamas, Phys. Rev. B 95, 214426 (2017).

[37] G. Vidal, J. I. Latorre, E. Rico, and A. Kitaev, Phys. Rev. Lett. 90, 227902 (2003).

[38] H. Bethe, Z. Phys. 71, 205 (1931).

[39] X. Plat, Y. Fuji, S. Capponi, and P. Pujol, Phys. Rev. B 91, 064411 (2015).

[40] C. A. Lamas, D. C. Cabra, P. Pujol, and G. L. Rossini, Eur. Phys. J. B 88, 176 (2015). 